\title{
Study on the of Fungi Antineoplastic Constituents in Root Plant of Acanthus ilicifolius $\mathbf{L}$
}

\author{
Wanke Zhang ${ }^{1}$, Dulin Kong ${ }^{1}$, Rui Yang ${ }^{2}$, Juan Li $^{{ }^{11}}$ \\ ${ }^{1 .}$ College of Pharmaceutical Sciences, Hainan Medicinal University, Haikou, 571199 China \\ 2.Hainan Fouth Health School, Haikou, 570311 China
}

Keywords: Acanthus ilicifolius L, fungi, anti-tumor, MTT method, pantropical mangrove plant, active ingredients.

\begin{abstract}
Acanthus ilicifolius L is a kind of pantropical mangrove plant. It is cold-natured and bland. The whole plant is officinal. According to recent studies, Acanthus ilicifolius L has a variety of pharmaceutical function, such as hepatoprotective effect, antioxidant, anti-tumor effect and insecticidal effect. To study the active ingredients of plant fungi of Acanthus ilicifolius L, we focus on the study of anti-tumor active ingredients. We studied the fermentation of hyphostroma of fungal strain LSJ120 in this laboratory which was isolated from the roots of Acanthus ilicifolius L. The ethanol extract, the extratable petroleum ether, the chloroform extract, the ethyl acetate extract, the n-butanol extract, the aqueous extract and 8 hyphostroma fermentation active constituents which was isolated from the ethyl acetate extract can be obtained by silica gel column chromatography. In Y1-Y8 component, 10 compounds were obtained from the separation and purification; Using MTT method, respectively, we do anti-tumor experiment for the extracts and compounds of each group. From the results, The inhibitive rate of the cells was the highest in the ethyl acetate extract. The highest value reached $80.73 \%$; from 8 kinds of components which were isolated from the ethyl acetate extract, the inhibition ratio of the Y6, Y7 components is significantly higher than the other groups; In addition, the compounds purified from the 8 kinds of isolates are also obtained correspondingly. The antineoplastic activity of compound extracted from Y6, Y7 components was stronger. Among them, the inhibition ratio of compound 6, compound 9 was significantly higher than other compounds, which is the main antineoplastic constituents of this fungi. By structure identification, the compound 6 was a cyclopentanone material, and the compound 9 is a cyclic dipeptide derivatives. The finding provides the scientific basis for the development and utilization of the medicinal value of Acanthus ilicifolius L.
\end{abstract}

\section{CLC number: R927 document code: A}

Lao shu le, Latin name is Acanthus illicifolius L. It is a kind of mangrove plant which grows in tropical coastal areas. It belongs to Acanthaceae. Its growing region is mainly distributed in tropical countries in Southeast Asia, such as China, India, Philippines, Malaysia, etc. It is mainly located in the southern coast and the littoral belt of China where the tides can arrive, such as Hainan, Guangdong, Guangxi, Fujian and Taiwan coastal areas, which is one of the important components of mangrove. According to the "Chinese herbal medicine compilation", the root and whole plant can be used as medicine. It has the effect of heat-clearing and detoxifying, reducing swelling and resolving mass, relieving cough and asthma. It is widely used as a specific drug for the treatment of hepatitis B (HBV) in folk . At present, the research of plant antitumoral natural products from fungi in root system of Acanthus ilicifolius $L$ has become the new field of marine medicine resources research and development [1].

In recent years, people's living environment is increasingly deteriorating, and pollution is increasingly serious. The incidence of human tumors has been rising year by year. The tumor also became one of the important diseases that harm mankind. According to statistics data, the tumor diseases kill millions of people every year all over the world. Even to this day, in the face of some tumor diseases, human beings are still helpless. The curative effect of traditional anti-viral drugs is low, even is invalid. So scholars So, the scholars urgently study the ways to conquer cancer disease 
completely.

Therefore, More and more scholars have turned their attention to the ocean in the industry. The ecological environment of halobios brings the unique genetic background, metabolic pathway and the diversity of species and particularity. It is likely to find the new antitumor and antiviral active substances, which provides the material resources for further development and treatment of new drugs of malignant tumors. At least $10 \%$ of the substances showed cytotoxic activity. According to the relevant reports in the USA, more than 1500 compound monomers were extracted from marine fungi by relevant scholars every year. According to the analysis, at least $1 \%$ of the compound monomers have antitumor activity[2-5]. Yang Junxian studied endogeny and rhizospheric microflora of soil in plant habitats of intertidal zone. The isolated bacteria were found from mangrove plants, which has strong antibacterial and insecticidal activity. The endophytic fungi has strong antitumor activity [6-8]. The research results of Yang Junxian Yang are similar to the results of the analysis of Acanthus ilicifolius $\mathrm{L}$ isolation and identification of rhizosphere endophytic bacteria by Wang Rong and Hong Kui. The antibacterial and insecticidal effects of bacteria isolated from endophytic bacteria were better than that of Actinomycetes [9-11].

From the research on Acanthus ilicifolius L root plant fungal antitumor active ingredient, after separation and purification, two kinds of antitumor substances were obtained. By structure identification, the compound 6 was a cyclopentanone material, and the compound 9 is a cyclic dipeptide derivatives. The finding provides the scientific basis for the development and utilization of the medicinal value of Acanthus ilicifolius L.

\section{Materials and methods}

\section{Drugs and instruments.}

VC7070E mass analyzer: Mass Spectrometry Instruments Ltd; PURIFY 100 medium pressure semi preparative chromatography; America CE Ltd; high performance liquid chromatograph: DIONEX; Bio-Rad 3550-UV ELIASA, Sanyo Corporation of Japan; SANYO MCO 15A CO2 incubator, the Sanyo Corporation of Japan; high speed centrifuge: Deutschland BECK MAN company; Rotary oscillation incubator: TCC100R (Takasaki the Scientific Instrument Corporation); CO2 incubator: MCO175 (SANYO); Vacuum concentration apparatus (Savant): SC250DDA;

Thin layer chromatography and column chromatography with silica gel: Qingdao marine chemical plant; fetal bovine serum: Lanzhou minhai biotechnology company, No. 20070424; petroleum ether, chloroform, ethyl acetate, butanol, ethanol and other reagents used are analytical reagent.

MTT activity test tumor cells is murine temperature sensitive type mammary cancer tsFT210cell; the fetal bovine serum is the product of Hyclone (Cat.No.STF721); DMSO cell culture medium was the product of Gibcobrl company;

Acanthus illicifolius L root plant fungal strain LSJ120 was isolated from the herbs plant with Acanthus ilicifofius roots, strains are preserved in the Wuhan University national culture preservation center.

\section{Experimental method}

\section{Fermentation and rough extraction.}

Mycelial fermentation: We put $100 \mathrm{ml}$ fluid medium in a $250 \mathrm{ml}$ erlenmeyer flask, and the $1 \mathrm{ml}$ fungus seed liquid are grown in the erlenmeyer flask, and then we put it into the table concentrator incubator. At $28^{\circ} \mathrm{C}, 220 \mathrm{RPM} / \mathrm{min}$, it was fermented for 5D.

The separation of active compounds: we take the extracts with strong anti-tumor activity to the silica gel column chromatography. Then we used dichloromethane and methanol (100: 0 to 0 :100) with gradient elution. The separation layer from Y1 to Y8 was obtained. The component with high inhibition rate was obtained by activity detection. After the separation of the reduplicated silica gel column chromatography, we use the UV lamp to detect the obvious highlights. The separation and purification of compounds as shown in table 1: 
Table 1 check list of active components and isolated compounds

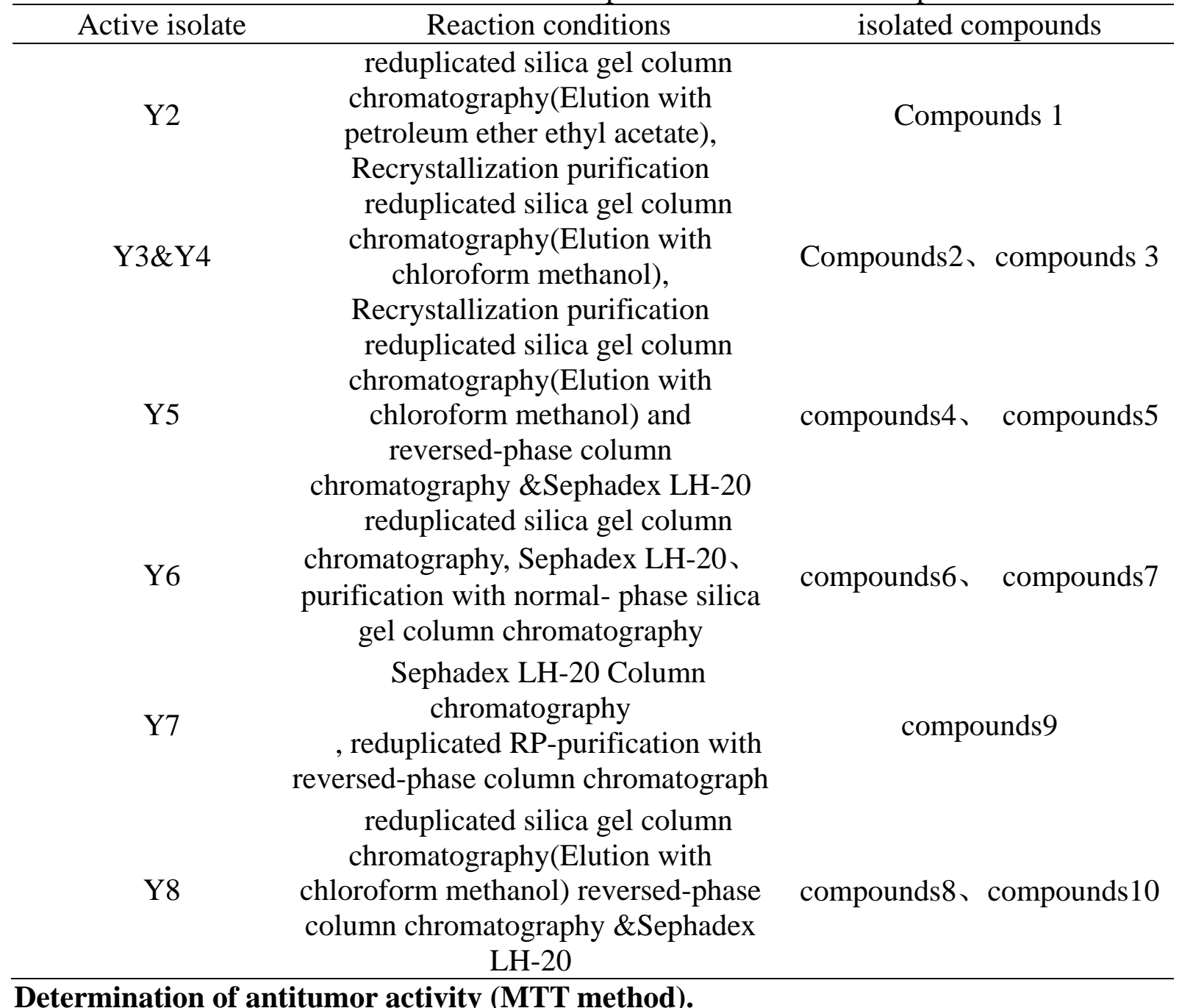

(1) The configuration of the test sample: respectively take the ethanol extracts of mycelium fermentation, the petroleum ether extract, chloroform extract, ethyl acetate extract, n-butanol extract and water extract, and the Isolated mycelium fermentation active compounds in ethyl acetate extract, active compounds, which is formulated as a $10000 \mathrm{mg} / \mathrm{l}$ stock solution by DMSO solution, and is saved at $4^{\circ} \mathrm{C}$.

(2) The cultivation of the tumor cells: a. configure the DMEM culture medium with $10 \%$ fetal bovine serum, penicillin streptomycin $100 \mathrm{u} / \mathrm{ml}$; b. the cells are at $37^{\circ} \mathrm{C}$ and $5 \% \mathrm{CO}$ gas incubator, change the culture medium for 2-3 days, cells had digestive transfer culture by using trypsin treatment.

\section{Data processing.}

Repeat 3 times for each group experiment, using DPS software for statistical analysis of experimental results. According to the formula, the inhibition rate is calculated, and we use Excel form mapping.

\section{Results and discussion}

\section{Inhibitory effects of different extracts on tumor cells.}

We used different components to react with tumor cells, respectively such as the ethanol extracts of mycelium fermentation, the petroleum ether extract, chloroform extract, ethyl acetate extract, n-butanol extract and water extract. 


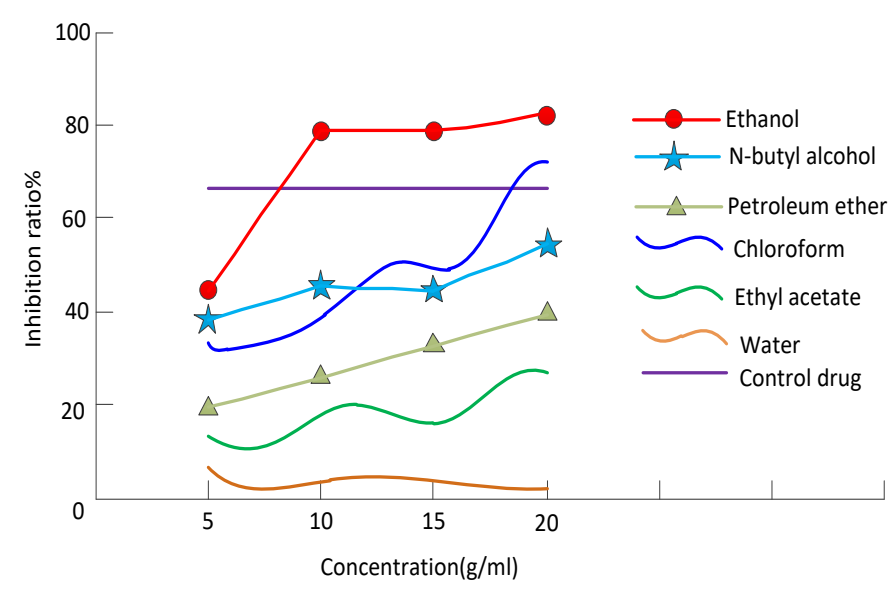

Fig. 1 inhibition rate of different extracts dispose mycelium fermentation to tumor cells

In Figure 1, we see the inhibition ratio of tumor cell with different components. From the figure 1 , the tumor cell inhibition rate of the ethyl acetate extract was highest. When the drug mass concentration was greater than $1 \mathrm{~g} / \mathrm{mL}$, the inhibition rate was greater than $77.84 \%$, and the highest value reached $80.73 \%$, which was significantly higher than the inhibition rate of control drug; the inhibition of other components: the n-butanol extract $>$ petroleum ether extract $>$ chloroform extract $>$ ethanol extract. The on tumor growth inhibiting rate of water extract was close to 0 . From the overall slope of the broken line, we can see that with the increase of the extract concentration, the cell inhibition rate is higher, that is to say, teh extract has obvious dependence on the growth inhibition of tumor cells and the concentration of the reagent.

Inhibition of different concentrations ethyl acetate handles mycelium fermentation active compound to tumor cells.

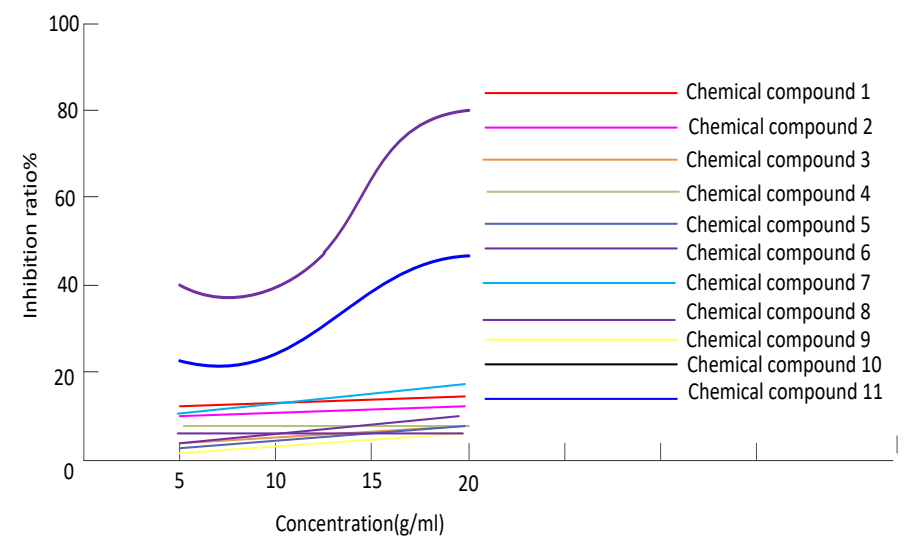

Fig. 2inhibition ratio of different concentrations ethyl acetate handles mycelium fermentation active compound to tumor cells

Figure2 shows the inhibition ratio of different concentrations ethyl acetate handles mycelium fermentation active compound to tumor cells, MTT inhibition rate shows that the anticancer activity of compound 6 and compound 9 is stronger, which is significantly higher than the other group. There is a good inhibitory effect on the growth of cancer cells. The inhibition ratio effect of compound 6 is the best. When the concentration is $10 \mathrm{~g} / \mathrm{mL}$, the inhibition rate of tumor cells is $97.45 \%$. Under the same concentration, its inhibition ratio is significantly higher than that of compound 7 which is isolated from Y6 component. So we infer that compound 6 is the main antitumor active substances of the ethyl acetate extract Y6 isolated fractions.

\section{Discussion}

In this paper, from the Acanthus ilicifolius L root plant fungal mycelia yeast, we can obtain the ethanol extract, the extratable petroleum ether, the chloroform extract, the ethyl acetate extract, the 
n-butanol extract, the aqueous extract, and 8 hyphostroma fermentation active constituents which was isolated from the ethyl acetate extract. In Y1-Y8 component, 10 compounds were obtained from the separation and purification; Using MTT method, respectively, we do anti-tumor experiment for the extracts and compounds of each group. From the results, The inhibitive rate of the cells was the highest in the ethyl acetate extract. The highest value reached $80.73 \%$; from 8 kinds of components which were isolated from the ethyl acetate extract, the inhibition ratio of the Y6, Y7 components is significantly higher than the other groups; In addition, the compounds purified from the 8 kinds of isolates are also obtained correspondingly. The antineoplastic activity of compound extracted from Y6, Y7 components was stronger. Among them, the inhibition ratio of compound 6, compound 9 was significantly higher than other compounds, which is the main antineoplastic constituents of this fungi. By structure identification, the compound 6 was a cyclopentanone material, and the compound 9 is a cyclic dipeptide derivatives. The finding provides the scientific basis for the development and utilization of the medicinal value of Acanthus ilicifolius L.

\section{Acknowledgements}

《Study on the antitumor constituents of mangrove plant Acanthus Illicifolius root 》,the National Natural Science Foundation of Hainan Province (No.20158362)

\section{References}

[1] XIE Li-sha, LIAO Yue-kui ,HUANG Quan-fang,et al.Pharmacognostic studies on mangrove Acanthus ilicifolius[J]. CHINA JOURNAL OF CHINESE MATERIA MEDICA,2005, 30(19):1501. [2] Teoh Y P. Antifungal activities of selected wood-degrading fungi of rubberwood.[J]. Journal of Tropical Forest Science, 2015, 27(3):325-333.

[3] Zhao Dan,Yu Lei , Chen Shouqian,et al.Chemical Constituents from the Roots of Acanthus Illicifolius[J]. Guangdong Chemical Industry,2015, 42(18):24-25.

[4] Xu xun .Marine Biotechnology and Sustainable Development of Marine Life Resources[J]. ENGINEERING SCIENCE, 2000, 2(8):40-42.

[5] Zhao D, Xie L, Yu L, et al. New 2-Benzoxazolinone Derivatives with Cytotoxic Activities from the Roots of Acanthus ilicifolius.[J]. Chemical \& Pharmaceutical Bulletin, 2015, 63(12):1087.

[6] Syed Ali M Y. Green synthesis of Silver nanoparticle by Acanthus ilicifolius mangrove plant against Armigeressubalbatus and Aedesaegypti mosquito larvae[J]. Heteroatom Chemistry, 2014, 24(1):43-52.

[7] Su H R, He H, Huang Q Z, et al. First report of black spot of Acanthus ilicifolius caused by Fusarium solani in China.[J]. Plant Disease, 2014, 98(10):140723135253000.

[8] WANG Rong, HONG Kui .Isolation and Activity Assessment of Rhizospheric and Symbiotic Microbes from Bear's-breech (Acanthus illicifolius)[J].JOURNAL OF MICROBIOLOGY,2007, 27(3):24-26.

[9] Tan D, Jiang C, Tao Y. Chemical Constituents of Acanthus ilicifolius[J]. Chemistry of Natural Compounds, 2016, 52(5):951-952.

[10] Koutrotsios G. Bioconversion of lignocellulosic residues by Agrocybe cylindracea and Pleurotus ostreatus mushroom fungi - assessment of their effect on the final product and spent substrate properties.[J]. Food Chemistry, 2014, 161(11):127-135.

[11] Sugimori T, Okawa T, Eguchi S, et al. ChemInform Abstract: The First Total Synthesis of (-)-Benzomalvin A and Benzomalvin B via the Intramolecular Aza-Wittig Reactions.[J]. Cheminform, 1998, 29(42):7997-8008. 